\title{
Assisted reproductive technology in reproductive medicine - possibilities and limitations
}

\author{
Marian Szamatowicz \\ Medical Institute of PWSliP, Lomza, Poland \\ Department of Reproduction and Gynecological Endocrinology, Medical University of Bialystok, Poland
}

\begin{abstract}
Infertility has become an increasingly common health problem and has been estimated to affect approximately $10 \%$ of women in the reproductive age. Due to its high prevalence, it has been deemed a social disease by the World Health Organization (WHO). The causes of infertility are numerous and vary from person to person. As for treatment, the three main therapeutic strategies include pharmacological therapy, surgical therapy - mostly endoscopy, and assisted reproductive technology (ART). Recent decades have witnessed great progress in ART, resulting in successful treatment of the previously untreatable cases, particularly in the field of fertility preservation, preimplantation screening for aneuploidy, uterine transplantations and mitochondrial replacement techniques as prevention against a number of severe diseases. Regardless, ART treatment does not guarantee pregnancy and live birth. The success rate is much smaller as compared to the failure rate, it being among its most important limitations. Embryo implantation is an extremely complex process and represents the most critical step of the reproduction process in humans. Attempts to evaluate endometrial receptivity and strategies for its correction have been discussed. The search for new effective predictors of an individual prognosis remains a crucial challenge for the contemporary reproductive medicine.
\end{abstract}

Key words: infertility, assisted reproductive technology (ART), possibilities of ART, limitations of ART, endometrial receptivity

Infertility constitutes a significant challenge for the contemporary reproductive medicine. According to the World Health Organization (WHO), infertility is a disease entity and, due to its prevalence, was deemed a social disease. Furthermore, it is considered as a specific and unique health problem because it always concerns two people simultaneously: a woman and a man. Individually, they can be in the best of mental and physical health but as a couple, who wishes to conceive and fails, they may face the problem of infertility. The desire to form a family and produce progeny is a natural human need and, as such, has been included in the Universal Declaration of Human Rights. International estimates on the prevalence of infertility and treatment seeking from 25 population surveys sampling 172413 women have demonstrated a $9 \%$ prevalence rate. Based on these estimates and the current world population, over 72 million women are currently infertile and over 40 million are currently seeking medical care [1]. More recent sources have reported that infertility affects as many as 186 million people worldwide, with male infertility contributing to over half of all cases of global childlessness [2]. Poland lacks detailed epidemiological data concerning infertility. Rough calculations have estimated that over one million couples are affected by infertility and approximately 200000 couples annually seek medical help. Noteworthy, stress which accompanies the diagnosis of infertility is on a par with the diagnosis of acute myocardial infarction or neoplastic disease. The only truly successful therapy of infertility includes pregnancy and live birth.

The therapy of infertility should precisely target the diagnosed cause of infertility, applying methods which guarantee the highest chance for pregnancy and delivery. At present, three main therapeutic strategies include pharmacological therapy, surgical therapy (preferably endoscopic techniques), and assisted reproductive technology (ART). Recent decades have witnessed great progress in ART, re- 
sulting in successful treatment of the previously untreatable cases. Regardless, social research to counterbalance the dominance of biomedical studies, is necessary, particularly with regard to expectations of infertile couples in ART [3].

In order to describe the current possibilities of ART in reproductive medicine, it seems prudent to briefly demonstrate the milestone achievements in that field. After the birth of Louise Brown in 1978 [4], other children were born in different countries, including Australia, the United States, France, and Sweden. Ultrasonographic techniques have been adopted to evaluate ovarian follicles [5], controlled ovarian hyperstimulation with clomiphene citrate, and with menopausal gonadotrophins has been introduced [5], agonist $\mathrm{GnRH}$ has been applied in stimulation protocols [6], oocyte retrieval has been performed under ultrasonographic guidance [7], and the first live birth after embryo freezing [8], as well as after ultra-rapid embryo freezing [9] have been reported. Significant progress in the treatment of the male factor of infertility was made in 1988, when pregnancy and live birth after microsurgical epididymal sperm aspiration (MESA) was reported [10], followed by live birth after intracytoplasmic sperm injection (ICSI) four years later [11]. In vitro maturation (IVM) of immature oocytes was another important step in the development of ART [12]. Since 1992, recombinant gonadotrophins have been introduced to the ovarian hyperstimulation protocols [13]. 1999 marked the beginning of the preimplantation diagnostic processes [14], and first live birth after biopsy of the blastocyst occurred three years later [15]. In 2004, the first baby after orthotropic transplantation of cryopreserved ovarian tissue was born [16], allowing for the development of fertility preservation methods.

The abovementioned 'milestones' in the field of reproductive medicine are merely some examples of ideas, their realization, and overwhelming progress in technology, which have accompanied the diagnosis and treatment of infertility. Still, these achievements constitute a suitable background for the presentation of the contemporary possibilities of ART.

Fertility preservation in young cancer patients as well as delayed motherhood, when the ovaries have been affected by the aging process, has been given much attention. Modern oncology has enabled over $80 \%$ of young people with cancer to survive. However, cancer treatment with potentially gonadotoxic therapies is often detrimental to the reproductive function. Patients or parents should be informed about fertility preservation options before treatment commencement using gonadotoxic therapy. Well-established and confirmed methods of fertility preservation include sperm cryopreservation for men and embryo or oocyte cryopreservation for women. Cryopreservation and transplantation of the ovarian cortex and testicular tissue remain to be investigated, with very promising results. Ovarian tissue can be easily obtained using laparoscopic techniques, without significant delay in gonadotoxic treatment [17]. Fertility preservation in women with cervical, endometrial and ovarian cancers is provided by both, fertility-sparing surgery and ART [18]. According to the literature, prevention of fertility in women with gynecological cancers is possible without disturbing the oncological safety [19].

The so-called 'absolute untreatable uterine factor', which affects $3-5 \%$ of the general population, is among the causes of infertility [20]. In cases when the affected woman rejects the idea of surrogacy or adoption, uterine transplantation remains the only solution. The development of surgical techniques and progress in transplantation immunology are the reasons behind the recent addition of a uterus to the list of organs successfully transplanted in humans [21]. Numerous animals, including rats, mice, rabbits, pigs, sheep and primates have been used as experimental models in the last decade. A significant number of attempts on humans has been performed in Turkey. Literature data revealed 11 subjects yielding positive pregnancy test but no live births. The first live birth after uterus transplantation took place in Sweden. Uterus transplantation is a very specific procedure and after the delivery of the child/children, the uterus has to be removed. Such method of treating absolute infertility is prudent only in situations when a blastocyst had been achieved and cryopreserved earlier. The procedure, although controversial, is an ethically acceptable solution in cases of absolute uterine infertility [21].

Advanced maternal age increases the chances of an aneuploid pregnancy and, as a result, the risk of producing an embryo with a genetic disease. Chromosomal defects also have an evident impact on the effectiveness of ART. Recently, a new generation of preimplantation genetic screening (PGS) tests has been introduced, the so-called PGS.2. Various aspects are extensively discussed, including the indications, the developmental stage, i.e. which material should be studied and which molecular techniques should be used [23, 24]. CRISP-Cas9 (clustered regularly interspaced short palindromic repeats with the molecular structure of enzyme Cas9) is the most advanced method of preimplantation diagnosis, allowing for prevention of mitochondrial diseases. Together with mitochondrial replacement therapy, it has become a promising tool to prevent transmission in patients with higher mutant mitochondrial loads $[25,26]$. While the fertilized egg inherits its nuclear DNA from both parents, the origin of mitochondrial DNA is exclusively maternal and in human reproduction it is associated with a broad range of transmitted debilitating and fatal disease. Mitochondrial replacement techniques have been successfully introduced as a solution to the problem. It is known as the'three-parent technique'. Although the treatment is legal in England and 
has been approved by the FDA in the US, it is the source of much controversy in many countries $[28,29,36]$.

The abovementioned tools of modern reproductive medicine with the use of ART, although sometimes generating spectacular results, are not without certain limitations. At present, there are commonly accepted measures of infertility treatment. A baby to take home or a live birth per cycle are the gold standards. Also, the safety measures in ART are not neglected or omitted. Still, the most significant limitation is the effect of therapy. A $25 \%$ rate of live births per cycle is believed to be a'good'success rate. Nevertheless, the $75 \%$ 'failure' rate remains a fact. Stress accompanying an ineffective procedure is extremely high, comparable to the initial diagnosis of infertility. The cost of the procedure is considerable and should not be ignored, especially in Polish conditions. Most recent data on the population of almost 180000 women have demonstrate the chance of a live birth after 3 complete cycles to be $42.3 \%$ [30], which is consistent with other publications. The chances for a live birth after 3, 6, and 12 cycles were $52 \%, 72 \%$ and $85 \%$, respectively, almost the same as in natural procreation [31]. The most limiting factor in an effective ART therapy is implantation failure, responsible for over $72 \%$ of all failures. Human implantation is a complex process, requiring synchrony between a healthy blastocyst and a functionally receptive endometrium. 'Endometrial receptivity' is described as temporary and exceptional sequence of events and factors which decide that an embryo can implant. The implantation takes place during the so-called 'endometrial window of implantation' (WOI), which lasts 4-5 days, with the general assumption that it starts 6-10 days after the ovulation and can be identified using morphological and biochemical markers [32, 33, 34]. In clinical practice, non-invasive methods of assessing endometrial receptivity are very much appreciated. Transvaginal ultrasonography 2/3D power Doppler has been proposed as a useful tool in the assessment of endometrial receptivity. It is possible to identify patients with bad prognosis by measuring the thickness and structure of the endometrium and also the endometrial and sub-endometrial blood flow $[32,35]$. As a result of much extensive research, new tests have been developed as molecular diagnostic tools. Such tests allow to identify delayed WOI as well as advanced WOI, allowing to personalize embryo transfer. Endometrial function test (EFT) evaluates endometrial expression of cyclin $E$ and p27, another one is based on the evaluation of MAG (a substance excreted by endometrial cell before the ovulation). Still, the research continues to develop a test based on a single molecular marker. The list of potential candidates is long, based on temporary expression of many proteins during the implantation window $[37,38]$.

When defective endometrial receptivity is diagnosed, strategies for improving the receptivity are proposed, mostly in cases with ART treatment. Four main strategies may be differentiated. First, development of ovarian stimulation protocols causing minimum reduction in endometrial receptivity, or even increasing it, is advised. Promising results have been reported when clomiphene citrate was not used in ovarian stimulation protocols or by vaginal supplementation of estradiol and progesterone gel $[32,39]$. Second, freezing of all embryos and performing embryo transfer in subsequent natural cycles are recommended to avoid the negative influence of the endometrium during stimulated cycles [40], but this strategy is not commonly appreciated [32]. Third, improvement of uterine vascularization by changing the uterine and the ovarian blood flow by treating patients with low dose aspirin, with nitric oxide donors like I-arginine, with intravaginal administration of sildenafil are mentioned $[32,41]$. Fourth, in order to eliminate all functionally and anatomically pathological conditions, treatment of the luteal phase defects by removal of fibroids from the uterine cavity or intrauterine adhesions, and of hydrosalpinx are suggested [32].

The literature offers numerous reports about prognostic factors associated with the outcomes of in vitro fertilization (IVF), such as maternal age and ovarian ageing, diagnosis, and the ovarian reserve. At the same time, relatively little attention has been paid to lifestyle and IVF outcomes. Smoking, alcohol consumption, bad nutritional habits, caffeine intake, exercise and exposure to toxic bisphenols are evidently associated with lower rates of IVF success [42]. Also, exposure to power-frequency magnetic field and its impact on reproductive health in humans needs further research as well.

ART has been the source of a number of successes, some of them rather spectacular, but the problem of higher efficacy is of the outmost importance. It is a well-known fact that not all infertile couples may be successfully treated, hence the discovery of new, precise 'predictors' of an individual prognosis has become the greatest challenge in the field of reproductive medicine.

\section{REFERENCES}

1. Boivin J, Bunting L, Collins JA, Nygren KG. International estimates of infertility prevalence and treatment-seeking: potential need and demand for infertility medical care. Hum Reprod. 2007, 22 (6), 1506.

2. Inhorn $\mathrm{Cl}$, Pasquale P. Infertility around the globe: new thinking on gender, reproductive technologies and global movements in the $21^{\text {st }}$ century. Hum Reprod Update. 2015, 21 (4), 411.

3. Kamel RM. Assisted Reproductive Technology after the Birth of Luise Brown. J Reprod Infertil. 2013, 14 (3), 96.

4. Steptoe PC, Edwards RG. Birth after re-implantation of human embryo. Lancet. 1978, 2 (8085), 366.

5. Cohen J, Trounson A, Dawson K, [et al.]. The early days of IVF outside the UK. Hum Reprod Update. 2005, 11 (5), 439.

6. Fleming R, Adam AH, Barlow DH, [et al.]. A new systematic treatment for infertile women with abnormal hormone profiles. Br J Obstet Gyneacol. 1982,89 (1), 80

7. Lenz S, Lauritsen JG. Ultrasonically guided percutaneous aspiration of human follicles under local anesthesia: a new method of collecting oocytes for in vitro fertilization. Fertil Steril. 1982, 38 (6), 673 
8. Trounson A, Mohr L. Human pregnancy following cryopreservation, thawing and transfer of an eight-cell embryo. Nature. 1983, 305 (5936), 707.

9. Trounson A, Peura A, Kirby C. Ultra-rapid freezing: a new low-cost and effective method of embryo cryopreservation. Fertil Steril. 1987, 48 (5), 843.

10. Patrizio P, Silber S, Ord T, Balmaceda JP, Asch RH. Two birth after microsurgical sperm aspiration in congenital vas deference. Lancet. 1988, 2 (8624), 1364.

11. Palermo G, Joris $H$, Devroey $P$, Van Steirteghem AC. Pregnancies after intracytoplasmic injection of single spermatozoon into an oocyte. Lancet. 1992, 340 (8810), 17.

12. Cha KY, Koo JJ, Ko JJ, Choi DH, Han SY, Yoon TK. Pregnancy after in vitro fertilization of human follicular oocytes collected from non-stimulated cycles, their culture in vitro and their transfer in a donor oocyte program. Fertil Steril. 1991, 55 (1), 109.

13. Devroy P, van Steirteghem A, Mannaerts B, Bennink HC. Successful in vitro fertilization and embryo transfer after treatment with recombinant FSH. Lancet. 1992, 339 (8802), 1170.

14. Xu K, Shi ZM, Veeck LL, Hughes MR, Rosenwaks Z. First unaffected pregnancy using preimplantation genetic diagnosis for sickle cell anemia. JAMA. 1999, 281 (18), 1701.

15. De Boer K, McArthur S, Murray C, Jansen RPS. First live birth following blastocyst biopsy and PGD analysis. Reprod Biomed Online. 2002, 4, 35.

16. Donnez J, Dolmans MM. Livebirth after orthotopic transplantation of cryopreserved ovarian tissue. Lancet. 2004, 364 (9443), 1405.

17. von Wolff M, Donnez J, Hovatta O, [et al.]. Cryopreservation and autotransplantation of human ovarian tissue prior to cytotoxic therapy - a technique in its infancy but already successful in fertility preservation. Eur J Cancer. 2009, 45 (9), 1547.

18. Feichtinger $M$, Rodriguez-Wallberg KA. Fertility preservation in women with cervical, endometrial and ovarian cancer. Gynecol Oncol Res Pract. 2016, 3, 8.

19. Zapardiel I, Cruz M, Diestro MD, Requena A, Garcia-Velasco JA. Assisted reproductive techniques after fertility-sparing treatments in gynecological cancers. Hum Reprod Update. 2016, 3.

20. Ozkan O, Dogan MU, Ozkan O, [et al.]. Uterus transplantation: from animal model through the first beating pregnancy to the first human birth. Wom Health. 2016, 12 (4), 442.

21. Testa G, Koon EC, Johanesson L. Living donor uterus transplant and surrogacy ethical analysis according to the principle of equipoise. Am J Transplant. 2016, 10, 1111.

22. Brannstrom $M$, Johannesson $L$, Bokström $H$, [et al.]. Livebirth after uterus transplantation. Lancet. 2015, 385 (9968), 607.

23. Geraedts J, Sermon K. Preimplantation genetic screening 2.0: the theory. Mol Hum Reprod. 2016, 22 (8), 839.

24. Sermon K, Capalbo A, Cohen J, [et al.]. The why, the how and when of PGS 2.0: current practices and expert opinions of fertility specialist, molecular biologist, and embryologist. Mol Hum Reprod. 2016, 22 (8), 845.

25. Vasenna R, Heindrycks B, Peco R, [et al.]. Genome engineering through CRISPR/Cas9 technology in the human germline and pluripotent stem cells. Hum Reprod Update. 2016, 22 (4), 411.
26. Fogleman S, Santana C, Bishop C, Miller A, Capco DG. CRISPR/Cas9 and mitochondrial gene re-placement therapy: promising techniques and ethical considerations. Am J Stem Cells. 2016, 5 (2), 39.

27. Babayev E, Seli E. Oocyte mitochondrial function and reproduction. Curr Opin Obstet Gynecol. 2015, 27 (3), 175.

28. Richardson J, Irving L. Concise reviews: Assisted reproductive technologies to prevent transmission of mitochondrial DNA disease. Stem Cells. 2015, 33 (3), 639.

29. Hyslop LA, Blakeley P, Craven L, [et al.]. Towards clinical application of pronuclear transfer to prevent mitochondrial DNA Disease. Nature. 2016, 534 (7607), 383

30. McLernon DJ, Maheshwari A, Lee AJ, Bhattacharya S. Cumulative live birth rates after one or more complete cycles of IVF: a population-based study of linked cycle data from 178898 women. Hum Reprod. 2016, 31 (3), 572.

31. Gnoth C, Maxrath B, Skonieczny T, Friol K, Godehardt E, Tigges J. Final ART success rates: a 10 years survey. Hum Reprod. 2011, 26 (8), 2239.

32. Einashar AM, Aboul-Einen GI. Endometrial Receptivity-Middle East. Fert Soc J. 2004, 9 (1), 10

33. Acosta AA, Elberger L. Endometrial dating and determination of the window of implantation in healthy fertile women. Fertil Steril. 2000, 75 (4), 788.

34. Achache $\mathrm{H}$, Revel A. Endometrial receptivity markers, the journal to successful embryo implantation. Hum Reprod Udate. 2006, 12 (6), 731.

35. Fanchin N. Assessing uterine receptivity in 2001: ultrasonographic glances in the new millennium. Ann NY Acad Sci. 2001, 943, 185.

36. Zhang J, Liu H, Luo S, [et al.]. First live birth using human oocytes reconstituted by spindle nuclear transfer for mitochondrial DNA mutation causing Leigh syndrome. Fertil Steril. 2016, 106 (3), e375.

37. Miravet-Valenciano JA, Rincon-Bertolin A, Vilella F, Simon C. Understanding and improving endometrial receptivity. Curr Opin Obstet Gynecol. 2015, 27 (3), 187.

38. Mahajan M. Endometrial receptivity array: clinical application (2015). J Hum Reprod Sci. 2015, 8 (3), 121.

39. Milachich T, Shterev A. Are there optimal numbers of oocytes, spermatozoa and embryos in assisted reproduction? JBRA Assist Reprod. 2016, 20 (3), 142.

40. Levran D, Dor J. Pregnancy potential of human oocytes. The effect of cryopreservation. Engl J Med. 1990, 323, 1153.

41. Chwalisz K, Garfield RE. Role of nitric acid in implantation and menstruation. Hum Reprod. 2000, 3, 96

42. Firns S, Cruzat VF, Keane KN, [et al.]. The effect of cigarette smoking, alcohol consumption and fruit and vegetable consumption on IVF outcomes: a review and presentation of original data. Reprod Biol Endocrinol. 2015, 13, 134.

43. Levis RC, Hauser R, Maynard AD, [et al.]. Exposure to Power-Frequency magnetic fields and the risk of infertility and adverse pregnancy outcomes: update on the human evidence and recommendations for future study designs. J Toxicol Environ Health B Crit Rev. 2016, 19 (1), 29. 